\title{
Mitigation of Speckle Noise in Laser Doppler Vibrometry by Using a Scanning Average Method
}

\author{
JINGHAO ZHU ${ }^{1,2,3}$, YANLU LI ${ }^{1,2, *}$, AND ROEL BAETS ${ }^{1,2}$ \\ ${ }^{1}$ Photonics Research Group, Ghent University-imec, Technologiepark-Zwijnaarde 126, 9052, Ghent, Belgium \\ ${ }^{2}$ Center for Nano- and Biophotonics (NB-Photonics), Ghent University, Technologiepark-Zwijnaarde 126, 9052, Ghent, Belgium \\ ${ }^{3}$ 1D-405 Science Park, Harbin Institute of Technology, 2 Yikuang Street, Harbin, 150080, China \\ *Corresponding author: Yanlu.Li@UGent.be
}

Compiled March 7, 2019

We present a scanning average (SA) method used in laser Doppler vibrometry (LDV) systems for mitigating the noise induced by dynamic speckles. In this method, the measurement beam is scanned over the target surface within the area of interest at a relatively high frequency. Then an averaging operation (e.g. low-pass filtering) is applied to the acquired photocurrent signals to remove the impacts of the scan. Movement signals recovered from the averaged photocurrents turn out to have lower speckle-induced noise. We report the experimental demonstration of this technique through the use of a silicon-based photonic integrated circuit (PIC). (C) 2019 Optical Society of America

OCIS codes: (120.3180) Interferometry; (030.6140) Speckle; (120.7280) Vibration analysis.

\section{http://dx.doi.org/10.1364/ao.XX.XXXXXX}

By offering non-contact measurements with a wide frequency response and a high spatial resolution, laser Doppler vibrometry (LDV) has become an increasingly popular technique for various fields [1-4]. Different from the piezoelectric accelerometers, LDV sensors avoid many problems in contact measurements, such as undesired mass-loading effects and difficulties in sensor installation [5]. One major problem with LDV is the dynamic speckles in the back-reflection [6], which usually lead to low signal-to-noise ratios (SNRs) in the output vibration signals [2]. The speckles originate from a target surface that is optically rough or treated with a retro-reflective tape in which the surface imperfections are on the order of the light wavelength. When a coherent light beam is incident on this surface, constructive or destructive interferences of the scattered rays lead to unpredictable bright or dark regions in the reflection field (speckle pattern). Unavoidable off-axial target motions, such as in-plane movement, cause a movement of the speckle patterns. These dynamic speckles lead to two major issues in LDV: (1) Phase noise: the phases of a bright speckles are inhomogeneous and randomly distributed in the space domain [2]. The phase variations associated to the speckles' movements can be transformed to the LDV outputs and thus cause the phase noise. (2) Spurious jumps: a dark speckle corresponds to a reflection with a low power. When

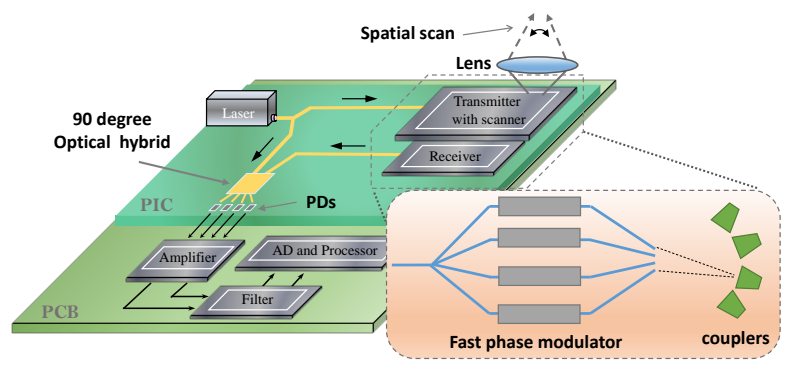

Fig. 1. The schematic of an example on-chip LDV system to implement the proposed SA method.

the dark speckles move across the receiving antennas (RAs), the demodulator may generate strong spurious phase jumps as a result of wrong interpretations of the weak reflections by the non-linear performance of the demodulator [7].

Mitigation methods to speckle noise in LDV systems have been investigated for many years. J. Vass et al reported that speckle noise can be detected by using an indicator and then removed by a time domain algorithm [2]. J. Aranchuk et al [8] developed and experimentally investigated methods to reduce the speckle noise in a multi-beam LDV. Polytec developed several despeckle methods, including a tracking filter method [9], a diversity combining method [10], and an adaptive optics method [11]. In this letter, we propose a novel method to reduce the impacts of dynamic speckle noise in homodyne LDV systems [12]. This method includes two steps. First, the measurement beam is actively scanned over a small area around the target with the help of a relatively fast optical scanner. As a result, the movement information of all locations in this area is obtained by the LDV. Then a low-pass filter is employed to achieve averaged photocurrent signals to ensure the impact from the scan is removed. The demodulation to the displacement signal is performed after the averaging step. With this scanning average (SA) method, the recovered signals experience much smaller noise caused by dynamic speckles. Compared to the previous speckle-mitigation methods, the SA method reduces the speckles only within the optical head of the LDV and ensures a simple post-processing. Detailed explanations and experimental demonstrations will be discussed in the following sections. 
To realize an LDV device with a proper SA process, it is necessary to use a scanner with a frequency much larger than the required bandwidth, e.g. $40 \mathrm{kHz}$ for a sound signal. This frequency is usually too high for a mechanical scanner. Therefore, a fast non-mechanical scanner is required, which can be realized with a PIC. The PIC also includes all necessary components for homodyne LDVs, e.g. optical splitters, photo-diodes (PDs), 90-degree optical hybrids [13], transmit-antennas (TAs) and RAs (see fig. 1). The scanner in the TAs can perform a spatial scan by using modulator-controlled arrayed waveguide gratings (AWGs) with a series of grating couplers (see fig. 1) and high-speed phase modulators with bandwidths up to $\mathrm{GHz}$ range [14]. The RAs should do the same scan as the TAs to enhance back-coupling efficiency. In practice, the TAs and RAs can share the same grating coupler designs. A stable laser source (wavelength $\lambda=1550 \mathrm{~nm}$ ) is connected to the PIC via a fiber or directly attached to the PIC with the help of a micro-optical bench [15]. Based on this design, we will explain the SA method.

Assume the displacement of a vibrating target is expressed as $d(t)=d_{V} \cdot \sin \left(2 \pi f_{m} t\right)$, where $t$ denotes the time, $f_{m}$ and $d_{V}$ are the frequency and the amplitude of the vibration, respectively. In the measurement, light sent to the PIC is firstly divided by a splitter to a measurement beam and a reference beam. The measurement beam is sent to the target via the TA and then reflected back to the RA. The optical system used to send and collect the measurement beam is an imaging system with a magnification of $M$, as is shown in fig. 2 (a), where $\xi$ and $x$ are the $2 \mathrm{D}$ coordinates in the target and source plane, respectively. The reflected electric field on the source plane is expressed as

$$
\boldsymbol{E}_{b}(\boldsymbol{x}, t)=\int \boldsymbol{E}_{t}(\boldsymbol{\xi}) \cdot a(\boldsymbol{\xi}) \cdot e^{i \Phi(\boldsymbol{\xi})} \cdot e^{i \phi_{\text {Doppler }}(t)} \cdot K(M \boldsymbol{x}-\boldsymbol{\xi}) d \boldsymbol{\xi},
$$

where $E_{t}(\boldsymbol{\xi})$ is the electric field incident on the target, $a(\boldsymbol{\xi})$ and $\Phi(\xi)$ are the surface-roughness-induced amplitude and phase change, respectively, $\phi_{\text {Doppler }}(t)$ is the Doppler phase of the useful signal, and $K(M x-\xi)$ is the point spread function of the optics in the backward (target to LDV) direction. To simplify the situation, we assume $a(\boldsymbol{\xi}) \equiv 1$. The field strength coupled to the RA is calculated by the field overlap of the reflection field and the receiver mode $\boldsymbol{E}_{r}(\boldsymbol{x})$ [16]:

$$
R(t)=\kappa \int e^{i \Phi(\xi)} \cdot C(\boldsymbol{\xi}) \cdot d \boldsymbol{\xi} \cdot e^{i \phi_{\text {Doppler }}(t)}
$$

where $\kappa$ is the normalizing constant of the field overlap, and $C(\boldsymbol{\xi})=\boldsymbol{E}_{t}(\boldsymbol{\xi}) \cdot \int \boldsymbol{E}_{r}^{*}(\boldsymbol{x}) \cdot K(M \boldsymbol{x}-\boldsymbol{\xi}) d \boldsymbol{x}$ is the back-coupling strength per reflection position, which is constant over time. $R(t)$ can be considered as a multiplication of two factors: the phasor of the useful Doppler shift $\exp \left[i \phi_{\text {Doppler }}(t)\right]$ and $A_{m}=$ $\kappa \int \exp [i \Phi(\xi)] \cdot C(\xi) d \xi$. When an off-axial translation introduces a dynamic speckle, $\Phi(\xi)$ should be changed to $\Phi(\xi, t)$ to include the factor of time. For example, when the target only has an off-axial translation $\boldsymbol{\xi}_{\text {oat }}(t), \Phi(\xi, t)=\Phi\left(\xi-\xi_{\text {oat }}(t)\right)$. This means both the phase and amplitude of $A_{m}(t)$ can vary as functions of time, which correspond to the two types of speckle noise mentioned in the first paragraph. In the PIC, this reflection is combined with the reference $A_{r}$ in the 90degree optical hybrid [12], and then detected by the PDs. With the help of differential amplifiers, two quadrature signals, i.e. $I(t)$ and $Q(t)$, are obtained, which can be considered as the real and imaginary parts of the complex photocurrent $S(t)=$ $4 \alpha A_{r} A_{m}(t) \exp \left[i \phi_{\text {Doppler }}(t)-i \theta_{s}\right]$, where $\alpha$ is the conversion efficiency combining photodiode responsivity with electronic amplification and $\theta_{S}$ is the constant phase difference. A common
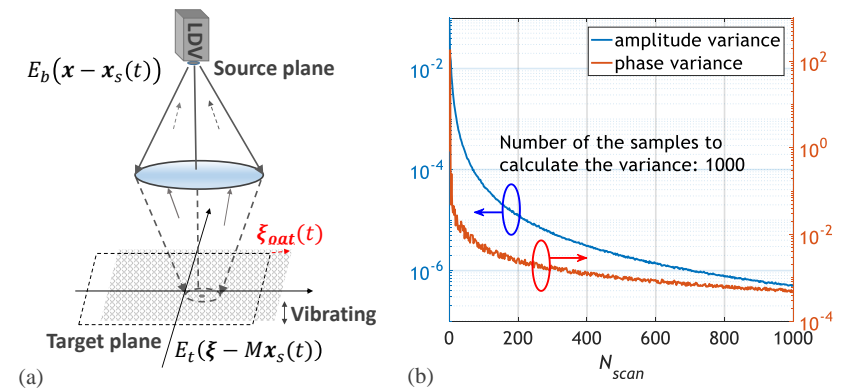

Fig. 2. (a) The backward field propagation in the imaging system. (b) The variance of $\left\langle\exp \left[i \Phi_{S}(\xi, t)\right]\right\rangle$ v.s. the number of scanned units.

method to obtain $d(t)$ is done by first retrieving $\phi_{\text {Doppler }}(t)$ from the arctan value of $Q(t) / I(t)$, and then converting the phase to the displacement by $d(t)=\phi_{\text {Doppler }}(t) \cdot \lambda / 4 \pi$.

To realize the SA method, a fast scan described by $x_{S}(t)$ is applied to the source field: $E_{0}\left(x-x_{s}(t)\right)$. As a result, the corresponding image becomes $E_{t}\left(\boldsymbol{\xi}-M x_{s}(t)\right)$. Since the field of the RA is also scanned as $\boldsymbol{E}_{r}\left(\boldsymbol{x}-\boldsymbol{x}_{s}(t)\right), A_{m}(t)$ becomes

$$
A_{m}(t)=\kappa \int e^{i \Phi_{s}(\xi, t)} \cdot C(\xi) \cdot d \xi
$$

where the phase change $\Phi_{S}(\xi, t)=\Phi\left(\xi+M x_{S}(t), t\right)$ is impacted by both the off-axial translation of the target and the scan of the laser beam. Note $C(\boldsymbol{\xi})$ remains constant over time thanks to the same scan in the TA and the RA. When the target only has an off-axial translation $\xi_{\text {oat }}(t), \Phi_{S}(\xi, t)=\Phi\left(\xi+M x_{S}(t)-\xi_{\text {oat }}(t)\right)$. The impact of the scan is removed by doing averaging to $S(t)$ :

$$
\langle S(t)\rangle=4 \alpha \kappa A_{r} e^{i \phi_{\text {Doppler }}(t)-i \theta_{s}} \int\left\langle e^{i \Phi_{s}(\xi, t)}\right\rangle \cdot C(\xi) \cdot d \xi .
$$

where $\langle\ldots\rangle$ denotes the time average with a finite time window which ensures the information of the displacement, i.e. $\exp \left[i \phi_{\text {Doppler }}(t)\right]$, is maintained while the signal variation related to the beam scanning is removed. This average can be realized by a low-pass filter with a proper cutoff frequency. Eq. 4 shows that the speckle-induced variance of the averaged $\langle S(t)\rangle$ is only determined by the variance of $\left\langle\exp \left[i \Phi_{S}(\xi, t)\right]\right\rangle$.

It can be numerically proven that the off-axial-translationinduced variance of $\left\langle\exp \left[i \Phi\left(\xi+M x_{s}(t)-\xi_{\text {oat }}(t)\right)\right]\right\rangle$ is reduced than that of $\exp \left[i \Phi\left(\xi-\xi_{\text {oat }}(t)\right)\right]$ (the case without the SA method) when the scanned region becomes larger. In the simulation, we assume the target surface comprises many coherent units with the same area and the same amplitude reflectance. By coherent unit, we mean that the reflection phase changes are the same across one coherent unit, but are totally random among different units. The scanned region includes $N_{i}$ units that are simultaneously illuminated by the sensing beam and $N_{\text {scan }}$ more units that are illuminated because of the beam scan. When an off-axial translation happens, $N_{\text {oat }}$ new coherent units are brought into this region while $N_{\text {oat }}$ units are moved out. Because the scan frequency is high, $N_{\text {oat }}$ can be considered unchanged during one scan cycle. Therefore the averaged phase-shift $\left\langle\exp \left[i \Phi\left(\xi+M x_{s}(t)-\xi_{\text {oat }}(t)\right)\right]\right\rangle$ can be numerically expressed as $w\left(N_{\text {scan }}, N_{\text {oat }}\right)=\sum_{k=N_{\text {oat }}}^{N_{i}+N_{\text {scan }}+N_{\text {oat }}} \exp \left(i \varphi_{k}\right) /\left(N_{i}+N_{\text {scan }}\right)$, where $\varphi_{k}$ is the random phase change for the $k$ th coherent unit. Here, the variation of $w\left(N_{\text {scan }}, N_{\text {oat }}\right)$ is caused by the random phases of the new coherent units. Fig. 2(b) shows both the amplitude and the phase variances of $w\left(N_{\text {scan }}, N_{\text {oat }}\right)$ as functions of $N_{\text {scan }}$ 


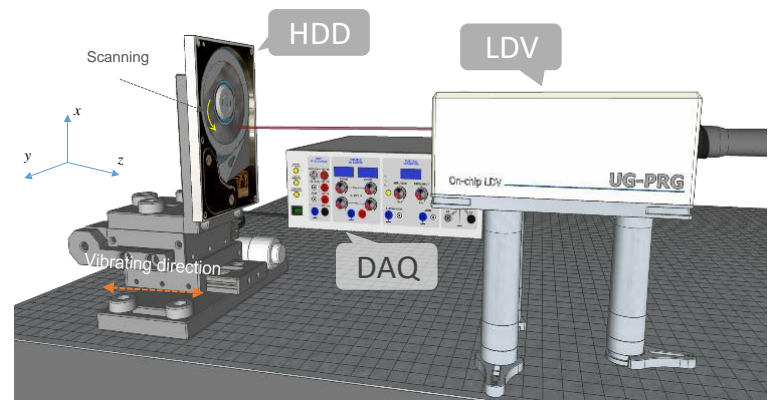

Fig. 3. The experimental setup for verifying the SA method.

for $N_{\text {oat }}=1$ and $N_{i}=2$. Here $N_{i}$ is not chosen as 1 to avoid a simulation error originated from the assumption that all coherent units have the same reflection amplitude. It is shown that both variances become smaller as $N_{\text {scan }}$ increases. The variances without the SA method are represented by the case for $N_{\text {scan }}=0$, which are larger than those with the SA method $\left(N_{\text {scan }}>0\right)$. This result also holds for $N_{o a t}>1$ or $N_{i}>2$, and thus validates our proposal method. A proper averaging time $\Delta t$ is selected based on two criteria. On one hand, $f_{\text {avg }}=1 / \Delta t$ should be greater than the bandwidth of the Doppler shift $B W_{\text {Doppler }} \approx 2 v_{m} / \lambda+f_{m}$ to ensure the vibration information is not averaged out, where $v_{m}$ is the target's maximum velocity. On the other hand, $f_{a v g}$ should be less than the minimal frequency generated by the scan $f_{\text {scan }}-B W_{\text {Doppler }}$ to ensure the impact of the scan is removed by the averaging, where $f_{\text {scan }}$ is the scan frequency. This is compatible with Carson's bandwidth rule [17].

The previous calculations imply that one can reduce the speckle impacts by increasing the number of reflectors, e.g. by enlarging the illuminated region, and then averaging the photocurrents before demodulation. A common way to enlarge the spot-size of the sensing beam is by increasing the magnification of the optical system. However, increasing the magnification also reduces the reflection strength since the numerical aperture (N.A.) of the reflection optics is decreased, which leads to a worse SNR in the LDV output. On the contrary, our method is based on a scan process, which ensures a good N.A. for the backcoupling while the averaging region is considerably increased. Therefore, the resultant SNR in the SA method is better than that obtained by increasing the magnification of the optical system. One note is that the scanned region should be small enough so that the vibration across the region is almost constant, but it should be large enough so that the scanned beam can walk across a sufficiently large number of scatters in each scan cycle.

To demonstrate this method experimentally, we use one PICbased homodyne LDV system with a rotating target to mimic the beam scanning. The setup is displayed in fig. 3. A harddisk drive (HDD) without a cover was fixed to a translation stage. Since there is no scanner for the laser beam, the disk of HDD rotated in the $x y$ plan to emulate the desired fast scan. The translation stage vibrated along the laser beam direction $(z$ direction) with the help of a piezoelectric actuator. Therefore, the entire HDD surface was considered to have the same vibration in the $z$ direction. Note that when the disk was rotating, there was a unavoidable wobbling at the rotation frequency. This also happens for a real scanning method, because the optical paths of a scanning beam may also have a small change during the scan. An on-chip homodyne LDV [18] was placed in the opposite side of the HDD to detect this vibration. A piece of micro-beads retro-

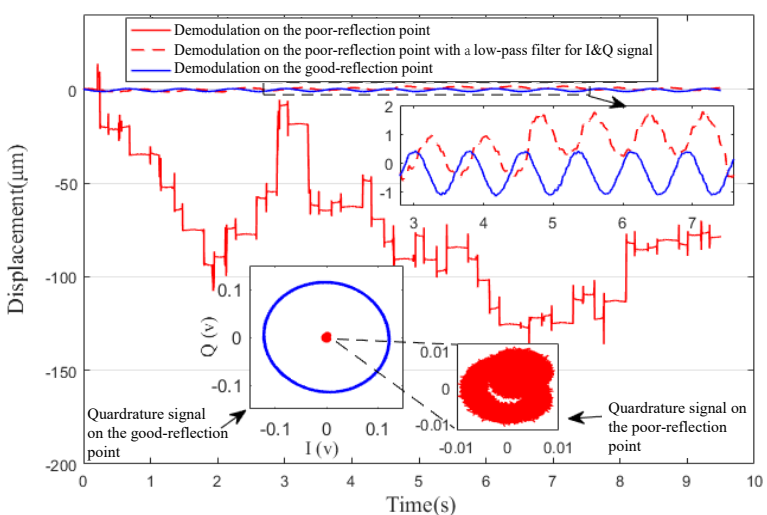

Fig. 4. The demodulated signals and the corresponding Lissajous curves of a normal good-reflection and a poor-reflection point.
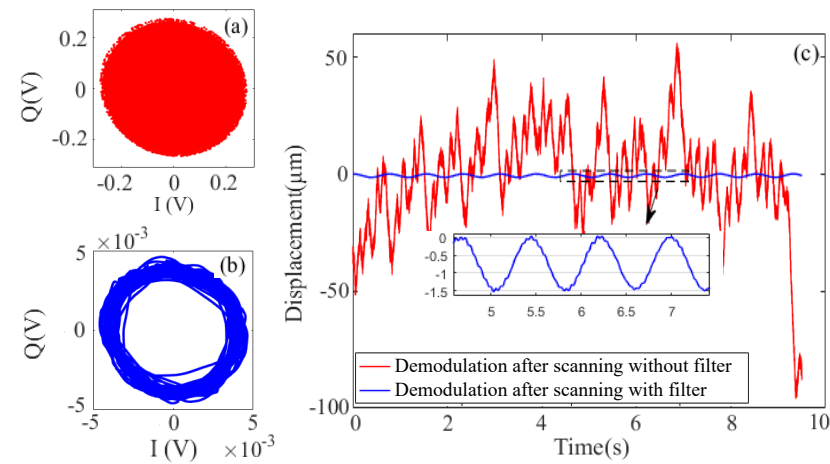

Fig. 5. Experimental results for a poor-reflection point: (a) and (b) show the Lissajous curves without and with a low-pass filter (cutoff frequency $=8.9 \mathrm{~Hz}$ ), respectively. (c) Demodulated results without (red) and with the filter (blue).

reflector ( $3 \mathrm{M} 7610$, glass-bead diameter $\approx 50 \mu \mathrm{m}$ ) was affixed on the whole area of the HDD disk. The measured location is around $20 \mathrm{~mm}$ away from the center. It is estimated that $N_{\text {scan }} \approx 8000$ and $N_{i} \approx 10$. All components were placed on a vibration-isolated optical table. A data acquisition card was used to collect the I\&Q signals. The filtering and demodulation processes were performed in the digital domain.

A sinusoidal vibration $\left(f_{m}=1.3 \mathrm{~Hz}, d_{V} \approx 0.72 \mu \mathrm{m}\right)$ was generated in $z$ direction without a rotation of the HDD. We used this low frequency mainly because we couldn't generate a high speed scanning with a normal mechanical method. To demonstrate the working principle, we chose to lower both $f_{\text {Doppler }}$ and $f_{\text {scan }}$. The position of the LDV was adjusted to ensure a good-reflection point (no speckle noise) and a poorreflection point (with speckle noise) of the retro-reflector were measured. As shown in fig. 4, the Lissajous trajectory of the $I \& Q$ signals for a good-reflection point was a nice circle, which means the SNR of the photocurrents was good. The corresponding demodulated displacement curve was smooth and without any jumping. By contrast, the $I \& Q$ signals obtained from the poorreflection point were very weak and the corresponding Lissajous curves had strong distortions. As a result, many obvious jumps appeared in the demodulated signal. These are the spurious jumps that we mentioned in the introduction part. Even when a low-pass filter with a cutoff frequency of $8.9 \mathrm{~Hz}$ was used in the 


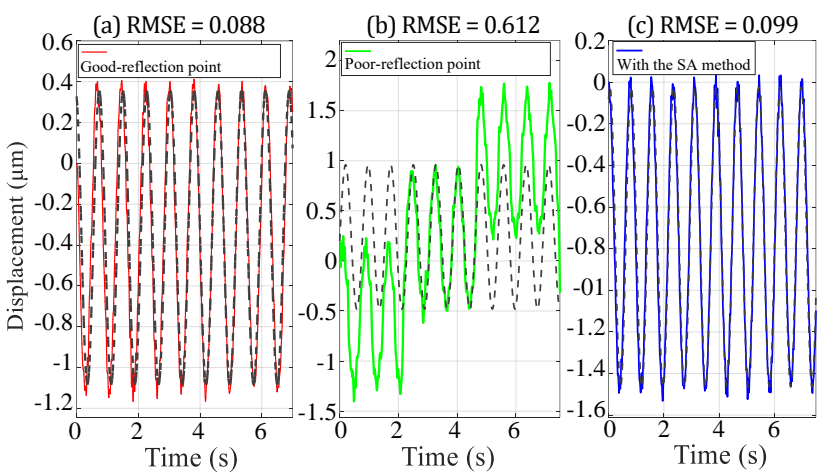

Fig. 6. Demodulated displacement for (a) the good-reflection point with I\&Q filtering, (b) the poor-reflection point with I\&Q filtering, and (c) the results obtained with the scanning average method. The dotted lines are the expected displacements.

$I \& Q$ signals to reduce noise, various spurious jumps still existed as is shown by the red dotted line in fig. 4 .

Then the SA method was tested by applying a $145 \mathrm{~Hz}$ rotation speed to the HDD. The Lissajous curve without averaging is shown in fig. 5 (a), which is very noisy due to the scan. As a result, the corresponding demodulated displacement signal, i.e. the red line in fig. 5(c), had strong scan-induced variations in the time domain. Then a low-pass filter with a cutoff frequency $f_{l p}$ was applied to the $I \& Q$ signals. Calculation shows that $B W_{\text {Doppler }}=8.9 \mathrm{~Hz}$. According to the aforementioned criteria, $f_{l p}$ was selected as $8.9 \mathrm{~Hz}$ to ensure a good SNR. Fig. 5 (b) shows a clear Lissajous circle after filtering, and the blue line in fig. 5 (c) shows a clean demodulated signal with an amplitude that is very similar to the input vibration amplitude $(0.72 \mu \mathrm{m})$. The quality of the three output signals was evaluated by the rootmean-square errors (RMSEs) between the demodulated signals and the expected displacement signals (with an amplitude of $0.72 \mu \mathrm{m}$ ). As is shown in fig. 6 , the measurement precision by using the SA method (RMSE $=0.099 \mu \mathrm{m}$ ) is close to that of a good-reflection point $(\mathrm{RMSE}=0.088 \mu \mathrm{m})$ and is obviously better than that of the poor-reflection point $(\mathrm{RMSE}=0.612 \mu \mathrm{m})$. These results prove that the SA method is effective to improve the quality of the signals affected by dynamic speckles.

A sweep of the cutoff frequency was made to prove the criteria of the averaging frequency. As is shown in fig. 7, the demodulated vibration amplitudes (green line) were stable and the corresponding SNRs (blue line) were high when $f_{l p}$ was in the range $\left(B W_{\text {Doppler }}, f_{\text {scan }}-B W_{\text {Doppler }}\right) \approx(8.9 \mathrm{~Hz}, 136.1 \mathrm{~Hz})$, which agreed to our previous proposal. In this region, more noise was filtered out as the cutoff frequency becomes smaller, which leads to a higher SNR for a smaller $f_{l p}$. Especially because a noise peaked at around $20 \mathrm{~Hz}$ exists in the vibration signal, the SNR in the frequency region between $10 \mathrm{~Hz}$ and $30 \mathrm{~Hz}$ are steeper than the rest part of the SNR curve.

In summary, a scanning average method was proposed in this letter to remove the speckle noise during vibration measurements. The experimental results show that the speckle-induced variations in the demodulated outputs (evaluated by the RMSEs) are considerably reduced with the help of the scanning average method. The corresponding SNRs are also improved. The experimental results are limited by the scanning speed of mechanical scanners. To realize a practical speckle mitigation for a signal with a bandwidth up to kilohertz range, a high speed scanner is needed. This is possible by using the high-speed on-chip phase

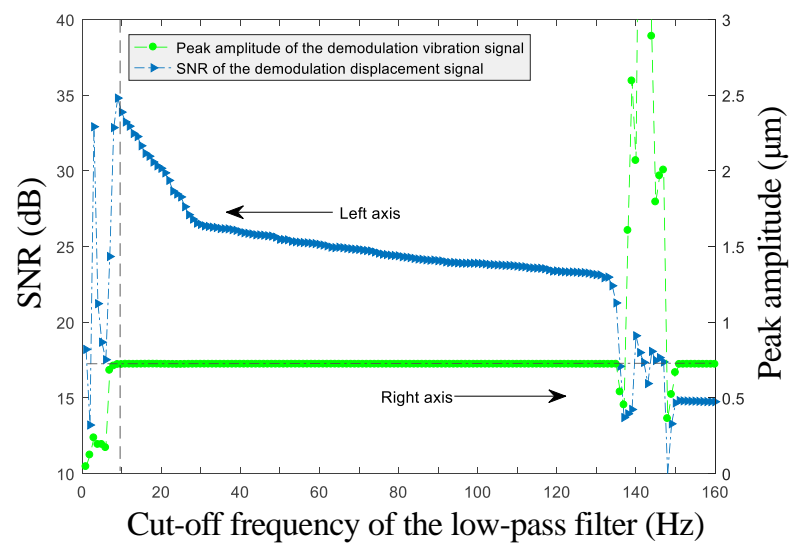

Fig. 7. The cutoff frequency of the low-pass filter v.s. the SNRs and the peak amplitudes of the demodulated signals.

modulators [14] which are well established in silicon-based photonic integrated circuits. With a proper design, the scanning frequency may reach hundreds of megahertz. In this case, the target can be considered as stationary in one scanning cycle (about several nanoseconds). Furthermore, the low-pass filter can be achieved directly in the photoelectric detection circuit, which makes the SA method perform more efficiently. Therefore, on-chip LDVs with fast scanners have the potential to realize a more practical scanning average method for speckle mitigation.

\section{FUNDING}

Methusalem grant "Smart Photonic Chips" of Prof. Roel Baets, Belgium; China Scholarships Council (CSC)(No.201606120142).

\section{REFERENCES}

1. M. S. Allen and M. W. Sracic, Mech. Syst. Signal Process. 24, 721 (2010).

2. J. Vass, R. Šmíd, R. Randall, P. Sovka, C. Cristalli, and B. Torcianti, Mech. Syst. Signal Process. 22, 647 (2008).

3. T. AbdElrehim and M. H. A. Raouf, MAPAN. 29, 1 (2014).

4. M. S. Allen, H. Sumali, and D. S. Epp, Nonlinear Dyn. 54, 123 (2008).

5. P. Castellini, M. Martarelli, and E. Tomasini, Mech. Syst. Signal Process. 20, 1265 (2006).

6. B. J. H. Steve J. Rothberg, Proc. SPIE 5503, 5503 (2004).

7. M. W. Sracic and M. S. Allen, In 27th Conf. Expo. on Struct. Dyn. (IMAC-XXVII Orlando USA) (2009).

8. V. Aranchuk, A. K. Lal, C. F. Hess, J. M. Sabatier, R. D. Burgett, I. Aranchuk, and W. T. Mayo, Proc. SPIE 6217, 621716 (2006).

9. D. Oliver, V. Palan, G. Bissinger, and D. Rowe, In 25th Conf. Expo. on Struct. Dyn. (IMAC-XXV Orlando USA) (2007).

10. A. Dräbenstedt, AIP Conf. Proc. 1600, 263 (2014).

11. C. Rembe and A. Dräbenstedt, AMA Conf. Sens. (2015).

12. Y. Li and R. Baets, Opt. Express 21, 13342 (2013).

13. R. Halir, G. Roelkens, A. Ortega-Moñux, and J. Wangüemert-Pérez, Opt. Lett. 36, 178 (2011).

14. H. Yu, W. Bogaerts, and A. De Keersgieter, IEEE J. Quantum Electron. 46, 1763 (2010).

15. M. Duperron, L. Carroll, M. Rensing, S. Collins, Y. Zhao, Y. Li, R. Baets, and P. O'Brien, Proc. SPIE 10109 (2017).

16. I. Awai and Y. Zhang, Microw. Conf. IEEE 363, 1223 (2015).

17. J. R. Carson, Proc. IEEE 51, 893 (1963).

18. Y. Li, J. Zhu, M. Duperron, P. O'Brien, R. Schüler, S. Aasmul, M. de Melis, M. Kersemans, and R. Baets, Opt. Express 26, 3638 (2018). 


\section{FULL REFERENCES}

1. M. S. Allen and M. W. Sracic, "A new method for processing impact excited continuous-scan laser doppler vibrometer measurements," Mech. Syst. Signal Process. 24, 721 - 735 (2010).

2. J. Vass, R. Šmíd, R. Randall, P. Sovka, C. Cristalli, and B. Torcianti, "Avoidance of speckle noise in laser vibrometry by the use of kurtosis ratio: Application to mechanical fault diagnostics," Mech. Syst. Signal Process. 22, $647-671$ (2008).

3. T. AbdEIrehim and M. H. A. Raouf, "Detection of ultrasonic signal using polarized homodyne interferometer with avalanche detector and electrical filter," MAPAN. 29, 1-8 (2014).

4. M. S. Allen, H. Sumali, and D. S. Epp, "Piecewise-linear restoring force surfaces for semi-nonparametric identification of nonlinear systems," Nonlinear Dyn. 54, 123-135 (2008).

5. P. Castellini, M. Martarelli, and E. Tomasini, "Laser doppler vibrometry: Development of advanced solutions answering to technology's needs," Mech. Syst. Signal Process. 20, 1265 - 1285 (2006).

6. B. J. H. Steve J. Rothberg, "Laser vibrometry meets laser speckle," Proc. SPIE 5503, 5503-5503- 12 (2004).

7. M. W. Sracic and M. S. Allen, "Experimental investigation of the effect of speckle noise on continuous scan laser doppler vibrometer measurements," In 27th Conf. Expo. on Struct. Dyn. (IMAC-XXVII Orlando USA) (2009).

8. V. Aranchuk, A. K. Lal, C. F. Hess, J. M. Sabatier, R. D. Burgett, I. Aranchuk, and W. T. Mayo, "Speckle noise in a continuously scanning multibeam laser doppler vibrometer for acoustic landmine detection," Proc. SPIE 6217, 621716 (2006).

9. D. Oliver, V. Palan, G. Bissinger, and D. Rowe, "3-dimensional laser doppler vibration analysis of a stradivarius violin," In 25th Conf. Expo. on Struct. Dyn. (IMAC-XXV Orlando USA) (2007).

10. A. Dräbenstedt, "Diversity combining in laser doppler vibrometry for improved signal reliability," AIP Conf. Proc. 1600, 263-273 (2014).

11. C. Rembe and A. Dräbenstedt, "D1.1 - speckle-insensitive laserdoppler vibrometry with adaptive optics and signal diversity," AMA Conf. Sens. (2015).

12. Y. Li and R. Baets, "Homodyne laser doppler vibrometer on silicon-oninsulator with integrated 90 degree optical hybrids," Opt. Express 21, 13342-13350 (2013).

13. R. Halir, G. Roelkens, A. Ortega-Moñux, and J. Wangüemert-Pérez, "High-performance 90 hybrid based on a silicon-on-insulator multimode interference coupler," Opt. Lett. 36, 178-180 (2011).

14. H. Yu, W. Bogaerts, and A. De Keersgieter, "Optimization of ion implantation condition for depletion-type silicon optical modulators," IEEE J. Quantum Electron. 46, 1763-1768 (2010).

15. M. Duperron, L. Carroll, M. Rensing, S. Collins, Y. Zhao, Y. Li, R. Baets, and P. O'Brien, "Hybrid integration of laser source on silicon photonic integrated circuit for low-cost interferometry medical device," Proc. SPIE 10109 (2017).

16. I. Awai and Y. Zhang, "Maxwell equations, coupled mode theory and coupling coefficient," Microw. Conf. IEEE 363, 1223-1225 (2015).

17. J. R. Carson, "Notes on the theory of modulation," Proc. IEEE 51, 893-896 (1963).

18. Y. Li, J. Zhu, M. Duperron, P. O’Brien, R. Schüler, S. Aasmul, M. de Melis, M. Kersemans, and R. Baets, "Six-beam homodyne laser doppler vibrometry based on silicon photonics technology," Opt. Express 26, 3638-3645 (2018). 